\title{
Mucormycosis: A Case Series of Patients Admitted in Non- COVID-19 Intensive Care Unit of a Tertiary Care Center during the Second Wave
}

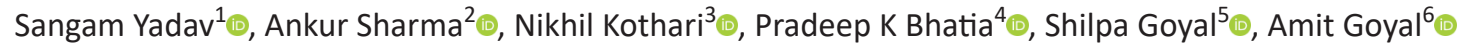

\begin{abstract}
Rhino-orbital-cerebral mucormycosis is an invasive fungal infection associated with mortality of $25-62 \%$. There has been a surge in the number of cases during this second wave of coronavirus disease-2019 (COVID-19) in India. We report 10 cases of mucormycosis admitted to our adult intensive care unit. After reviewing the patient's information, we found that $60 \%$ of patients had received steroids, and most had uncontrolled blood sugar levels. Most patients received treatment with surgical debridement and antifungal, although the mortality rate was as high as $40 \%$. We report two unique cases of renal and gastrointestinal mucormycosis as well. We concluded that poor glycemic control was the primary etiology behind the rise in the number of cases. Our report also stresses the importance of early surgical intervention and suggests further research comparing the efficacy of combination antifungal therapy versus single antifungal (amphotericin B) to help resource-limited settings in these times of drug crisis.
\end{abstract}

Keywords: COVID-19, Intensive care unit, Mucormycosis.

Indian Journal of Critical Care Medicine (2021): 10.5005/jp-journals-10071-23986

\section{INTRODUCTION}

Mucormycosis is a rare but potentially life-threatening infection caused by a fungus belonging to the Mucoraceae family. Generally, the susceptible population includes patients who have malignancy, are on steroids, are immune-compromised, are uncontrolled diabetic, etc. ${ }^{1}$ The annual incidence of mucormycosis is estimated to range from 1.7 cases per 1,000,000 inhabitants in the United States to 140 cases per $1,000,000$ in India and Pakistan. ${ }^{2}$ There has been a sudden surge in the number of mucormycosis cases during the second wave of coronavirus disease-2019 (COVID-19) in India. ${ }^{3}$ The most common site involved in Mucor infection is the paranasal sinus and orbit, leading to pain, swelling, numbness, and visual defect. However, renal and gastrointestinal involvement has also been reported though rarely. ${ }^{4,5}$ Here, we present a case series of 10 patients who were admitted to our adult ICU for mucormycosis management.

\section{Case Series}

We collected information of the patients admitted to our adult ICU, who were either diagnosed with mucormycosis or found to have the same latter in the course of treatment. The data were recorded over a period of 1 month from May to June, where we recorded the demographic information, comorbidities, h/o COVID-19 infection, dependency on steroid, evidence of mucormycosis, treatment received, duration between symptoms and surgery, length of ICU stay, and outcome (Table 1). The mean age of the study group was 47.5 years, ranging from 31 to 65 years. Forty percent were female and $60 \%$ males. Out of the 10 patients, 4 patients had COVID-19 infection, whereas 7 patients were recently diagnosed or known diabetic. Except for one patient who was a case of chronic liver disease (CLD), all the patients had uncontrolled sugar as shown by the HbA1c levels (glycosylated hemoglobin). Including the four patients who were earlier COVID-19
1,3-5 Department of Anaesthesia and Critical Care, All India Institute of Medical Sciences, Jodhpur, Rajasthan, India

${ }^{2}$ Department of Trauma and Emergency, All India Institute of Medical Sciences, Jodhpur, Rajasthan, India

${ }^{6}$ Department of Otorhinolaryngology, All India Institute of Medical Sciences, Jodhpur, Rajasthan, India

Corresponding Author: Ankur Sharma, Department of Trauma and Emergency, All India Institute of Medical Sciences, Jodhpur, Rajasthan, India, Phone: +91 9654045653, e-mail: ankuranaesthesia@ gmail.com

How to cite this article: Yadav S, Sharma A, Kothari N, Bhatia PK, Goyal S, Goyal A. Mucormycosis: A Case Series of Patients Admitted in Non-COVID-19 Intensive Care Unit of a Tertiary Care Center during the Second Wave. Indian J Crit Care Med 2021;25(10):1193-1196.

Source of support: Nil

Conflict of interest: None

positive, two more patients received steroid therapy; one was a case of rheumatoid arthritis. The other was given steroid because of suspicion of COVID-19 as radiologically, and the picture was of viral pneumonia in a local hospital. The mean duration of steroid therapy was 11 days. The most common presenting symptom was facial swelling. The mean duration between COVID symptoms and symptoms of mucormycosis was approximately 21 days. One patient presented with unique complaints of abdominal pain, decreased urination, and burning micturition and was diagnosed with renal mucormycosis on a biopsy of the kidney. One more patient presented with abdominal pain, vomiting, and constipation who was retrospectively found to have abdominal mucormycosis on histopathological examination (HPE). Most patients (8) required surgery and received liposomal amphotericin 


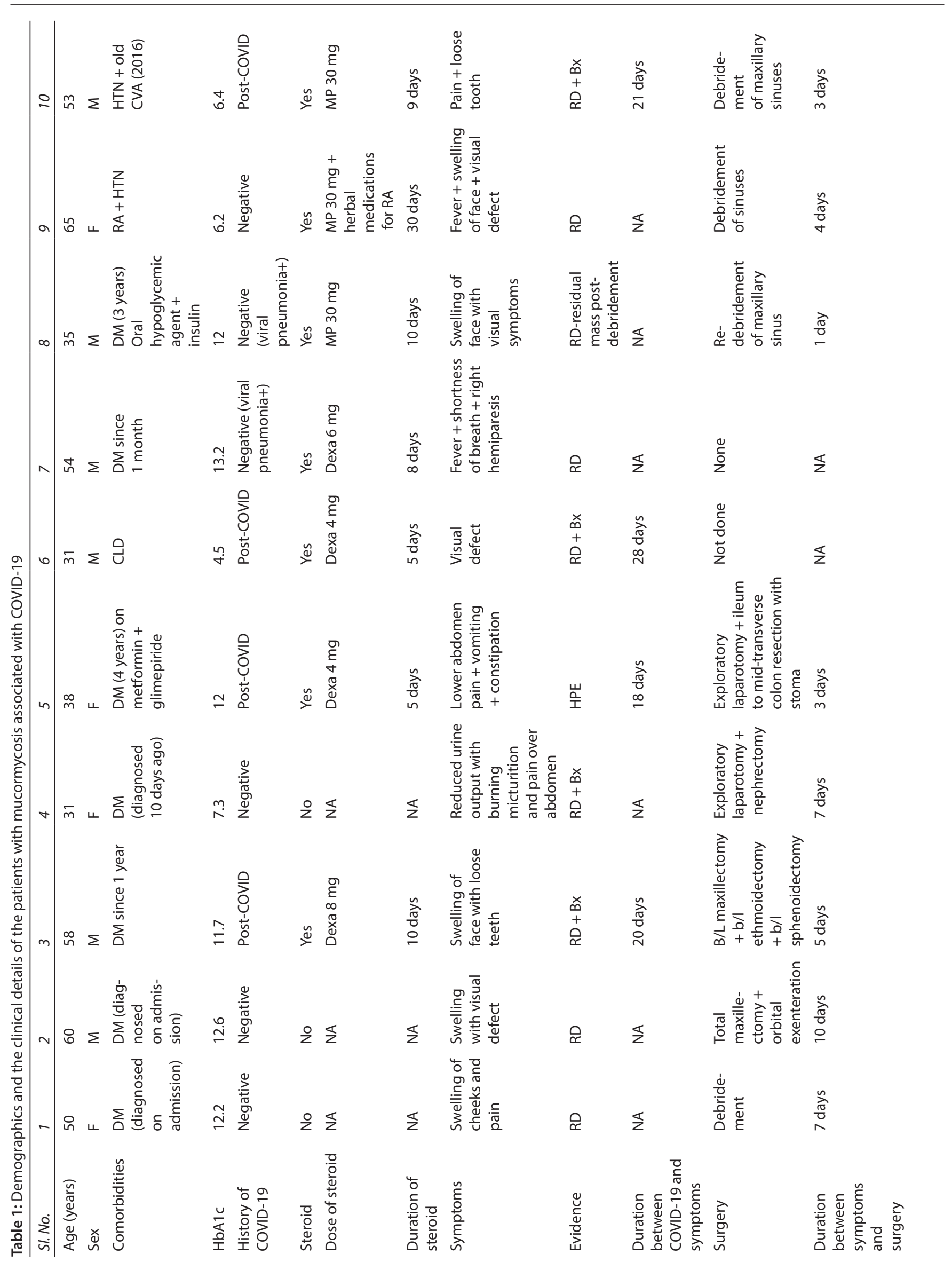




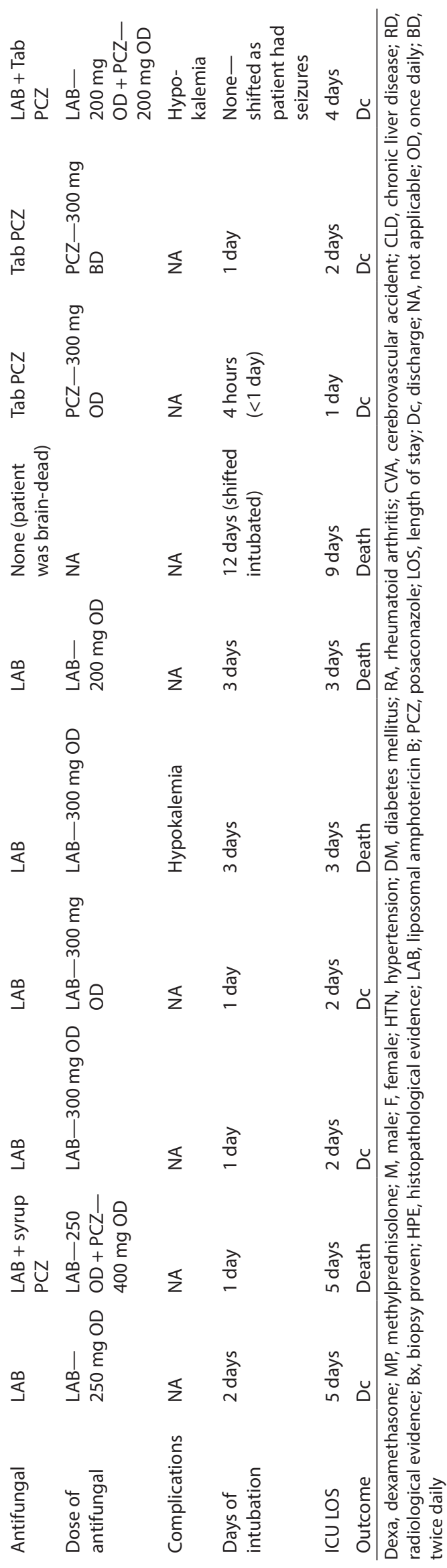

B either alone or in combination with posaconazole, except for two patients who received tablet posaconazole. Antibiotics were administered in accordance with the ICU protocol and culture sensitivity. Surgery could not be done for one patient with CLD who was hemodynamically unstable and the other patient who became brain-dead. Out of 10 patients, 4 succumbed to their illness, while the 6 were discharged; hence, the mortality was $40 \%$, which is similar to figures reported by Mishra et al. ${ }^{2}$

\section{Discussion}

Rhinocerebral mucormycosis is associated with a mortality rate of $25-60 \% .^{6}$ During the second wave of the COVID-19 pandemic, which India is going through, there has been a surge in the number of mucormycosis cases, and it has been made a notifiable disease in many states, including Rajasthan. The predisposing factors like diabetes mellitus, diabetic ketoacidosis, malignancy, immunocompromised state, and steroid therapy can lead to flaring infection caused by this fungus due to the lack of adequate chemotactic response/neutropenia/monocytes. The use of steroid therapy in the treatment of COVID-19 as advocated by the RECOVERY trial can be one factor for this surge. But multiple unevaluated factors like:

- Uncontrolled sugars and

- The use of industrial oxygen cylinders during this oxygen can also be contributing factor.

- Pro-coagulable state of the patient in COVID-19.

The disease is more common in males, as seen in our case series as well. ${ }^{7}$ Out of the 10 patients studied, 6 patients had received steroids, which could be the etiology for immunosuppression, but what is glaring is the uncontrolled sugars in all but 1 patient, which would have made them susceptible to the fungus. In addition, one patient with normal $\mathrm{HbA1c}$ levels was a patient with CLD, which in itself is an immune-suppressed state, thus predisposing to the infection.

We also report two unique cases of renal and intestinal mucormycosis. The etiology for the involvement is the endothelial invasion of the vessels by the fungus leading to thrombosis and infarction of the tissue. The most common route of spread for renal mucor is hematogenous, whereas the intestinal once can be due to ingestion or hematogenous both. ${ }^{8}$ In the gastrointestinal tract, the stomach and colon are commonly involved, as seen in our patient who had distal ileal and colonic gangrene. ${ }^{5}$ Isolated renal mucormycosis is rare and is generally bilateral, which has a very poor prognosis. ${ }^{9}$ High index of suspicion and early renal biopsy could help in early diagnosis and surgical intervention. As seen in our patient, the early surgical intervention helped in avoiding a catastrophe. Similarly, intestinal mucormycosis has a high mortality, as it is associated with multi-organ failure, which was seen in our patient too who succumbed to the illness.

There was an early surgical intervention in all patients except two (CLD with hemodynamic instability and brain-dead patient). The mean duration from symptoms to intervention was 5 days (ranging from 1 to 10 days). Early surgical intervention with early initiation of therapy with liposomal amphotericin B helped keep mortality to $40 \%$, including the two patients who did not receive surgical intervention. Excluding them, the mortality figure was $20 \%$.

One more problem arising now is the shortage of supply of amphotericin $B$ because of a sudden surge in the number of cases. In such cases, oral/i.v. formulation of posaconazole can be tried. We do 
not suggest it as first-line treatment, but in case of scarcity and after careful individualization in less critical individuals, posaconazole can be given as a trial. In our study, two of our patients who received posaconazole did well and were discharged from ICU by day 1 and day 2 , respectively. The other option can be a combination of amphotericin $B$ and posaconazole, which has similar efficacy but helps in reducing the dose of amphotericin $\mathrm{B} .^{10}$

\section{CONCLUSION}

- Early surgical debridement and initiation of antifungal therapy help in reducing mortality.

- Regular monitoring of blood glucose levels during COVID-19 treatment should help prevent mucormycosis infection.

- In the case of a resource-limited setting, consideration for a combination of amphotericin B and posaconazole, if amphotericin in less supply.

- A high index of suspicion of mucormycosis in susceptible patients with symptoms of pyelonephritis and obstruction, especially in the current scenario.

- Monthly audit in all ICUs to see the trend of mucormycosis and generate data.

\section{Future Scope}

A multicenter randomized control trial on the efficacy of amphotericin+posaconazole combination on mucormycosis is required. The possible duration post-COVID-19, when an individual is at the highest risk of acquiring mucormycosis infection, should be examined.

Also, the role of uncontrolled sugars in post-COVID-19 patients and industrial cylinder usage in the surge of mucormycosis cases need to be studied.

\section{ORCID}

Sangam Yadav (1) https://orcid.org/0000-0001-7329-8782

Ankur Sharma ㄴ) https://orcid.org/0000-0001-9339-6988
Nikhil Kothari 니 https://orcid.org/0000-0002-9829-905X

Pradeep K Bhatia @ https://orcid.org/0000-0001-5082-7151

Shilpa Goyal ৫ https://orcid.org/0000-0002-8983-0953

Amit Goyal @ https://orcid.org/0000-0002-4339-7541

\section{References}

1. Kauffman CA, Malani AN. Zygomycosis: an emerging fungal infection with new options for management. Curr Infect Dis Rep 2007;9(6):435-440. DOI: 10.1007/s11908-007-0066-4.

2. Prakash $\mathrm{H}$, Chakrabarti A. Global epidemiology of mucormycosis. J Fungi 2019;5(1):26. DOI: 10.3390/jof5010026.

3. Mishra N, Mutya VSS, Thomas A, Rai G, Reddy B, Mohanan AA, et al. A case series of invasive mucormycosis in patients with COVID19 infection. Int J Otorhinolaryngol Head Neck Surg 2021;7(5):867-870. DOI: 10.18203/issn.2454-5929.ijohns20211583.

4. Mathew G, Arumugam V, Murugesan S, Duhli N, Agarwal I. Renal mucormycosis: a rare cause of urinary tract infection leading to endstage renal disease (ESRD). J Trop Pediatr 2019;65(4):405-408. DOI: 10.1093/tropej/fmy059.

5. Monte Junior ESD, Santos MELD, Ribeiro IB, Luz GO, Baba ER, Hirsch $\mathrm{BS}$, et al. Rare and fatal gastrointestinal mucormycosis (zygomycosis) in a COVID-19 patient: a case report. Clin Endosc 2020;53(6):746-749. DOI: 10.5946/ce.2020.180

6. Balai E, Mummadi S, Jolly K, Darr A, Aldeerawi H. Rhinocerebral mucormycosis: a ten-year single centre case series. Cureus 2020;12(11):e11776. DOI: 10.7759/cureus.11776.

7. Lee FY, Mossad SB, Adal KA. Pulmonary mucormycosis: the last 30 years. Arch Intern Med 1999;159(12):1301-1309. DOI: 10.1001/ archinte.159.12.1301

8. Riley TT, Muzny CA, Swiatlo E, Legendre DP. Breaking the mold: a review of mucormycosis and current pharmacological treatment options. Ann Pharmacother 2016;50(9):747-757. DOI: 10.1177/1060028016655425.

9. Sathe KP, Mehta KP. Irreversible fatal renal failure resulting from isolated renal mucormycosis. Saudi J Kidney Dis Transpl 2014;25(6):1312-1314. DOI: 10.4103/1319-2442.144298.

10. Rodríguez MM, Serena C, Mariné M, Pastor FJ, Guarro J. Posaconazole combined with amphotericin $B$, an effective therapy for a murine disseminated infection caused by Rhizopus oryzae. Antimicrob Agents Chemother 2008;52(10):3786-3788. DOI: 10.1128/AAC.00628-08. 